\title{
SLIP AND HALL EFFECTS ON THE PERISTALTIC PUMPING OF A LINEAR VISCOELASTIC FLUID THROUGH A POROUS MEDIUM IN A CHANNEL
}

\author{
NUNNA MAHESH BABU ${ }^{1} \&$ S. SREENADH ${ }^{2}$ \\ ${ }^{I}$ Department of Mathematics, Arignar Anna Govt. Arts and Science College, Karaikal, Puducherry, India \\ ${ }^{2}$ Department of Mathematics, S V University, Tirupati, Andhra Pradesh, India
}

\begin{abstract}
In this paper, we studied the effects of Hall and slip on the peristaltic flow of a Jeffrey fluid through a porous medium in a two-dimensional channel under the long wavelength approximation. Closed form solutions are obtained for axial velocity and axial pressure gradient. The effects of various emerging parameters on the axial pressure gradient and pumping characteristics are discussed with the help of graphs.
\end{abstract}

KEYWORDS: Jeffrey Fluid, Slip, Porous Medium, Hall \& Peristalsis

Received: Jun 08, 2020; Accepted: Jun 28, 2020; Published: Sep 01, 2020; Paper Id.: IJMPERDJUN20201003

\section{INTRODUCTION}

The Jeffrey model is relatively simpler linear viscoelastic model using time derivatives instead of convicted derivatives for example the Oldroyd-B model does, it represents a rheology different from the Newtonian. Hayat et al. (2006) have discussed the effect of an endoscope on the peristaltic pumping of a Jeffrey fluid. Hayat et al. (2007) have investigated the peristaltic flow a Jeffrey fluid in an axisymmetric tube. The effect of an endoscope and magnet field on the peristaltic flow involving Jeffrey fluid have discussed by Hayat et al. (2008).

In addition, flow through a porous medium has been of significant attention in recent years predominantly amid geophysical fluid dynamicists. Examples of natural porous media are rye bread, the human lung, beach sand, stand stone, limestone, wood, bile duct, gall bladder with stones and in small blood vessels. Hayat et al. (2008) have studied the peristaltic flow of a Jeffrey fluid through a porous medium in a channel under the effect of magnetic field with compliant walls. Subba Reddy and Prasnath Reddy (2010) have investigated the effect of variable viscosity on peristaltic flow of a Jeffrey fluid through a porous medium in a planar channel.Jyothi et al. (2016) have studied the hyperbolic tangent fluid flow through a porous medium in an inclined channel with peristalsis. Ranjitha and Subba Reddy (2018) have discussed the radiation effect on the peristaltic flow of a Jeffrey fluid through a porous medium in a channel.

Hayat et al. (2007) studied the Hall effects on peristaltic flow of a Maxwell fluid in a porous medium. AboEldahab et al. (2010) haveinvestigated the effects of Hall and ion-slip currents on magnetohydrodynamic peristaltic transport and couple stress fluid.Gad (2014) has studied the effects of Hall current on peristaltic transport with compliant walls. Eldabe (2015) have studied the Hall Effect on peristaltic flow of third order fluid in a porous medium with heat and mass transfer. Effect of hall and ion slip on peristaltic blood flow of Eyring Powell fluid in a non-uniform porous channel was studied by Bhatti et al. (2016). Recently SubbaNarasimhudu and Subba Reddy (2017) have investigated the Hall effects on the peristaltic pumping of a Newtonian fluid in a planar channel. 
The peristaltic transport of a Newtonian fluid through a 2D micro channel where the slip effect is present was investigated by Kwang (2000). El Sehaway et al. (2006) have studied the effect of slip on the peristaltic flow of a Maxwell fluid in a channel. The effects of slip and non- Newtonian parameters on the peristaltic flow of a third grade fluid in a circular cylindrical tube were investigated by Ali et al. (2009). Chaube et al. (2010) have studied the slip effects on the peristaltic flow of a micropolar fluid in a channel. Effects of slip and induced magnetic field on the peristaltic flow of pseudoplastic fluid were analyzed by Noreen et al. (2011). Subba Reddy et al. (2012) have investigated the slip effects on the peristaltic motion of a Jeffrey fluid through a porous medium in an asymmetric channel under the effect of magnetic field. Slip effects on peristaltic transport of a Prandtl fluid in a channel under the effect of magnetic field was studied by Jyothi et al. (2015).

In view of these, we studied the effects of Hall and slip on the peristaltic flow of a Jeffrey fluid through a porous medium in a two-dimensional channel under the long wavelength approximation. Closed form solutions are obtained for axial velocity and axial pressure gradient. The effects of various emerging parameters on the axial pressure gradient and pumping characteristics are discussed with the help of graphs.

\section{MATHEMATICAL FORMULATION}

We consider the peristaltic motion of a Jeffrey fluid through a porous medium in a two-dimensional symmetric channel of width $2 a$ under the effect of magnetic field. The flow is generated by sinusoidal wave trains propagating with constant speed $c$ along the channel walls. A uniform magnetic field $B_{0}$ is applied in the transverse direction to the flow. The magnetic Reynolds number is considered small and so induces magnetic field neglected. Fig. 1 represents the physical model of the channel.

The geometry of the wall surface is defined as

$$
Y=H(X, t)=a+b \cos \frac{2 \pi}{\lambda}(X-c t)
$$

where $b$ is the wave amplitude and $\lambda$ is the wave length.

We shall carryout this investigation in a co-ordinate system moving with wave speed, in which the boundary shape is stationary. The co-ordinates and velocities in the laboratory frame $(X, Y)$ and the wave frame $(x, y)$ are related by

$$
x=X-c t, y=Y, u=U-c, v=V, p(x)=P(X, t) .
$$




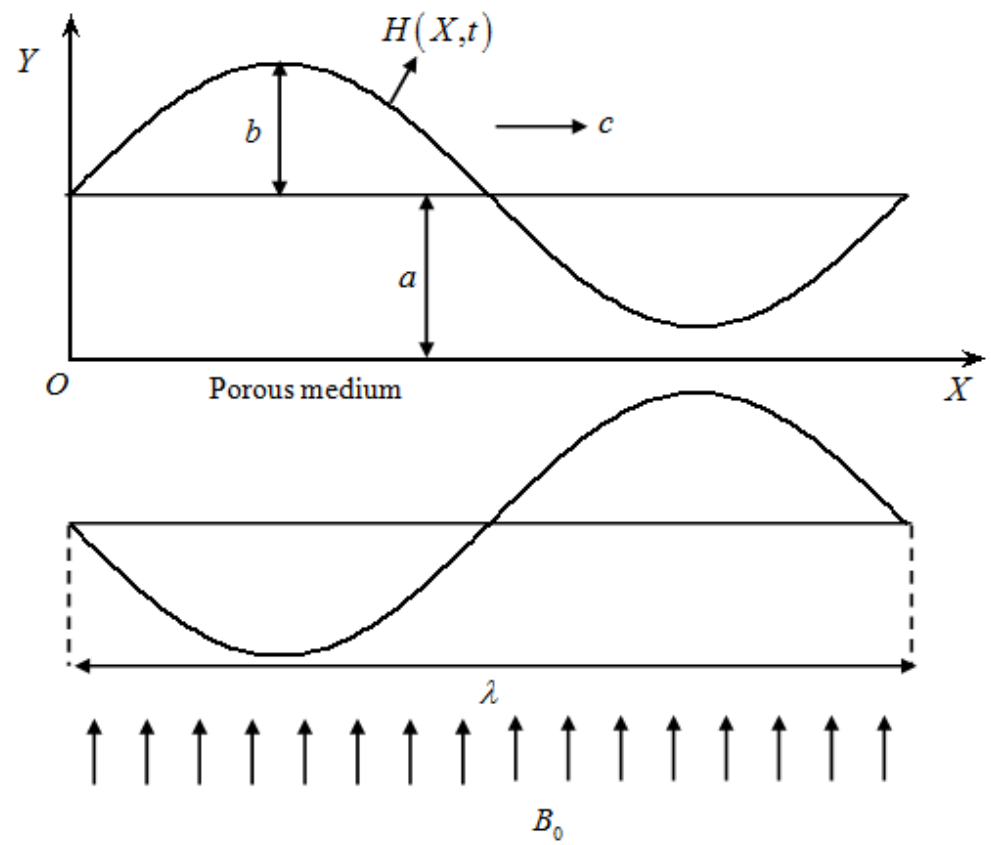

Figure 1: The Physical Model.

The equations governing the flow in the wave frame are

$\frac{\partial u}{\partial x}+\frac{\partial v}{\partial y}=0$

$\rho\left(u \frac{\partial u}{\partial x}+v \frac{\partial u}{\partial y}\right)=-\frac{d p}{d x}+\frac{\partial \tau_{x x}}{\partial x}+\frac{\partial \tau_{y x}}{\partial y}+\frac{\sigma B_{0}^{2}}{1+m^{2}}(m v-(u+c))-\frac{\mu}{k}(u+c)$

$\rho\left(u \frac{\partial v}{\partial x}+v \frac{\partial v}{\partial y}\right)=-\frac{d p}{d y}+\frac{\partial \tau_{x y}}{\partial x}+\frac{\partial \tau_{y y}}{\partial y}-\frac{\sigma B_{0}^{2}}{1+m^{2}}(m(u+c)+v)-\frac{\mu}{k} v$

where $\rho$ is the density $\sigma$ is the electrical conductivity, $B_{0}$ is the magnetic field strength and $m$ is the Hall parameter.

The constitute equation for the Jeffery fluid is

$$
\tau=\frac{\mu}{1+\lambda_{1}}\left(\dot{\gamma}+\lambda_{2} \ddot{\gamma}\right)
$$

where $\lambda_{1}$ is the ratio of relaxation time to retardation time, $\lambda_{2}$ is the retardation time, $\mu$ the dynamic viscosity, $\dot{\gamma}$ is the shear rate and dots over the quantities indicate differentiation with respect to time $t$.

The corresponding dimensional boundary conditions are

$u+\beta \tau_{x y}=-c \quad$ at $\quad y=H(x)$ (slip condition)

$\frac{\partial u}{\partial y}=0$ at $\quad y=0$ (symmetry condition)

The constitute equation for the Jeffery fluid is 
$\tau=\frac{\mu}{1+\lambda_{1}}\left(\dot{\gamma}+\lambda_{2} \ddot{\gamma}\right)$

using the following non-dimensional variables

$$
\bar{x}=\frac{x}{\lambda}, \bar{y}=\frac{y}{a}, \delta=\frac{a}{\lambda}, \bar{\mu}=\frac{u}{c}, \bar{t}=\frac{c t}{\lambda}, \bar{v}=\frac{v}{c \delta}, \bar{\tau}=\frac{a \tau}{\mu c}, \bar{p}=\frac{p a^{2}}{\mu c \lambda}
$$

in the equations (2.4) and (2.5), we get

$$
\begin{aligned}
& \operatorname{Re} \delta\left(u \frac{\partial u}{\partial x}+v \frac{\partial u}{\partial y}\right)=-\frac{d p}{d x}+\delta \frac{\partial \tau_{x x}}{\partial x}+\frac{\partial \tau_{y x}}{\partial y}+\frac{M^{2}}{1+m^{2}}(m \delta v-(u+1))-\frac{1}{D a}(u+1) \\
& \operatorname{Re} \delta^{3}\left(u \frac{\partial v}{\partial x}+v \frac{\partial v}{\partial y}\right)=-\frac{d p}{d x}+\delta^{2} \frac{\partial \tau_{x y}}{\partial x}+\delta \frac{\partial \tau_{y y}}{\partial y}-\frac{\delta M^{2}}{1+m^{2}}(m(u+1)+\delta v)-\frac{\delta^{2}}{D a} v
\end{aligned}
$$

where $\operatorname{Re}=\frac{r a c}{m}$ is the Reynolds number, $M=a B_{0} \sqrt{\frac{\sigma}{\mu}}$ is the Hartmann number,

$$
\begin{aligned}
& \tau_{x x}=\frac{2 \delta}{\left(1+\lambda_{1}\right)}\left[1+\frac{\lambda_{2} c \delta}{a}\left(u \frac{\partial}{\partial x}+v \frac{\partial}{\partial y}\right)\right] \frac{\partial u}{\partial x}, \\
& \tau_{x y}=\frac{1}{\left(1+\lambda_{1}\right)}\left[1+\frac{\lambda_{2} c \delta}{a}\left(u \frac{\partial}{\partial x}+v \frac{\partial}{\partial y}\right)\right]\left(\frac{\partial u}{\partial y}+\delta^{2} \frac{\partial v}{\partial x}\right), \\
& \text { And } \tau_{y y}=\frac{2 \delta}{\left(1+\lambda_{1}\right)}\left[1+\frac{\lambda_{2} c \delta}{a}\left(u \frac{\partial}{\partial x}+v \frac{\partial}{\partial y}\right)\right] \frac{\partial v}{\partial y} .
\end{aligned}
$$

Since we are interested in the case of inertia free, long wavelength as in Shapiro et al. (1969) and thus equations (2.11) and (2.12) reduces to

$$
\begin{aligned}
& \frac{1}{\left(1+\lambda_{1}\right)} \frac{\partial^{2} u}{\partial y^{2}}-\left(\frac{M^{2}}{1+m^{2}}+\frac{1}{D a}\right) u=\frac{\partial p}{\partial x}+\left(\frac{M^{2}}{1+m^{2}}+\frac{1}{D a}\right) \\
& \frac{\partial p}{\partial y}=0
\end{aligned}
$$

Equation (2.14) implies that $p$ is independent $y$, hence $p$ is only function of $\mathrm{x}$. Therefore, the Equation (2.13) can be rewritten as

$$
\frac{1}{\left(1+\lambda_{1}\right)} \frac{\partial^{2} u}{\partial y^{2}}-\left(\frac{M^{2}}{1+m^{2}}+\frac{1}{D a}\right) u=\frac{d p}{d x}+\left(\frac{M^{2}}{1+m^{2}}+\frac{1}{D a}\right)
$$

The corresponding non-dimensional slip boundary conditions in the wave frame are given by

$$
u+\frac{\beta}{1+\lambda_{1}} \frac{\partial u}{\partial y}=-1 \quad \text { at } \quad y=h=1+\phi \cos 2 \pi x
$$




$$
\frac{\partial u}{\partial y}=0 \quad \text { at } \quad y=0
$$

The volumetric flow ratio $q$ in the wave frame is given by

$$
q=\int_{0}^{h} u d y
$$

The instantaneous volume flow rate in the fixed frame is given by

$Q(x, t)=\int_{0}^{h} u d y=\int_{0}^{h}(u+1) d y=q+h$.

The time average flux over one period $T\left(=\frac{\lambda}{c}\right)$ of the peristaltic wave is

$\bar{Q}=\frac{1}{T} \int_{0}^{T} Q d t=q+1$

\section{SOLUTION}

Solving Equation (2.15) using boundary conditions (2.16) and (2.17) we get

$u=\left(\frac{1+\lambda_{1}}{\alpha^{2}}\right) \frac{d p}{d x}\left[c_{1} \cosh \alpha y-1\right]-1$

where $\alpha=\sqrt{\left(1+\lambda_{1}\right)\left(\frac{M^{2}}{1+m^{2}}+\frac{1}{D a}\right)}, c_{1}=\frac{1}{\cosh \alpha h+\beta_{1} \sinh \alpha h}$ and $\beta_{1}=\frac{\alpha \beta}{1+\lambda_{1}}$.

The volume flow rate $q$ in a wave frame of reference is given by

$$
q=\frac{1+\lambda_{1}}{\alpha^{3}} \frac{d p}{d x}\left[c_{1} \sinh \alpha h-\alpha h\right]-h
$$

From Eq. (3.2), we write

$$
\frac{d p}{d x}=\frac{(q+h) \alpha^{3}}{c_{1} \sinh \alpha h-\alpha h}
$$

The pressure rise over the one wave length of the peristaltic wave is given by

$$
\Delta p=\int_{0}^{1} \frac{d p}{d x} d x
$$

As $\lambda_{1} \rightarrow 0, D a \rightarrow \infty, M \rightarrow 0, m \rightarrow 0$ and $\beta \rightarrow 0$ our results coincides with results of Shapiro et al. (1969).

\section{DISCUSSIONS OF THE RESULTS}

In order to perceive the effects of variousfactors like Jeffrey fluid parameter $\lambda_{1}$, Darcy number $D a$, Hall parameter $m$, 
Hartmann number $M$, slip parameter $\beta$ and amplitude ratio $\phi$ on the pressure gradient we have plotted figure 2 to figure 7. From figure 2 , it is found that the axial pressure gradient $\frac{d p}{d x}$ decreases with increasing $\lambda_{1}$. From figure 3 , it is observed that, the axial pressure gradient $\frac{d p}{d x}$ decreases on increasing $D a$. From Fig. 4 , it is observed that the axial pressure gradient $\frac{d p}{d x}$ decreases with an increase in $m$. From figure 5 it is seen that the axial pressure gradient $\frac{d p}{d x}$ increases as increasing $M$. From figure 6 , it is noticed that the axial pressure gradient $\frac{d p}{d x}$ decreases on increasing $\beta$. From Fig. 7it is observed that, the axial pressure gradient $\frac{d p}{d x}$ increases with an increase in $\phi$.

In order to see the effects of various factors like Jeffrey fluid parameter $\lambda_{1}$, Darcy number $D a$, Hall parameter $m$ , Hartmann number $M$, slip parameter $\beta$ and amplitude ratio $\phi$ on pumping characteristics we have plotted Fig. 8 to figure 13.

From figure 8 , it is observed that the time-averaged flow rate $\bar{Q}$ decreases in both the pumping $(\Delta p>0)$ and free-pumping $(\Delta p=0)$ regions with increasing $\lambda_{1}$, while it increases in the co-pumping region $(\Delta p<0)$ with increasing $\lambda_{1}$. From Fig. 9, it is observed that the time-averaged flow rate $\bar{Q}$ decreases in the pumping region with increasing $D a$, while it increases in both the free-pumping and co-pumping regions with increasing $D a$.From figure 10, it is observed that, the time-averaged flow rate $\bar{Q}$ decreases in the pumping region with increasing $m$, while it increases in both the free-pumping and co-pumping regions with increasing $m$. From figure 11, it is found that the time-averaged flow rate $\bar{Q}$ increases in the pumping region with increasing $M$, while it decreases in both the free-pumping and co-pumping regions with increasing $M$. From figure 12it is noted that, the time-averaged flow rate $\bar{Q}$ decreases in both the pumping and freepumping regions with increasing $\beta$, while it increases in the co-pumping regions with increasing $\beta$ for chosen $\Delta p(<0)$. From figure 13,it is found that the time-averaged flow rate $\bar{Q}$ increases in both the pumping and free-pumping regions with increasing $\phi$, while it decreases in the co-pumping regions with increasing $\phi$ for chosen $\Delta p(<0)$.

\section{CONCLUSIONS}

In this paper, we studied the effects of Hall and slip effects on the peristaltic pumping of a Jeffrey fluid through a porous medium in a two-dimensional channel under the assumption of long wavelength. The expressions for the velocity field and axial pressure gradient are obtained analytically. It is found that, the axial pressure gradient $\frac{d p}{d x}$ and the time-averaged flow rate $\bar{Q}$ in the pumping region are increases with increasing $M$ and $\phi$, whereas they decreases with increasing $\lambda_{1}, m$ and $\beta$. Further, it is found that the pumping is more for Newtonian fluid $\left(\lambda_{1} \rightarrow 0\right)$ than that of Jeffrey fluid. 


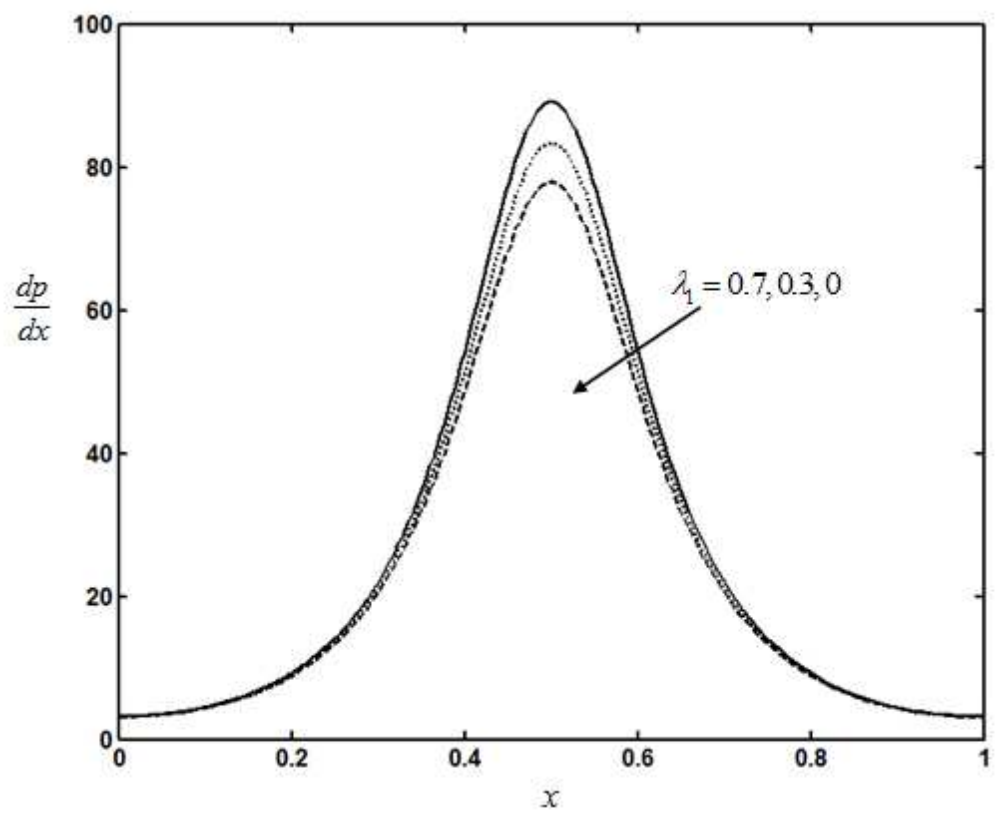

Figure 2: The Variation of Axial Pressure Gradient $\frac{d p}{d x}$ with Jeffrey

Fluid Parameter $\lambda_{1}$ for $\bar{Q}=-1, M=1, D a=0.1, \phi=0.6, \beta=0.1$ and $m=0.3$.

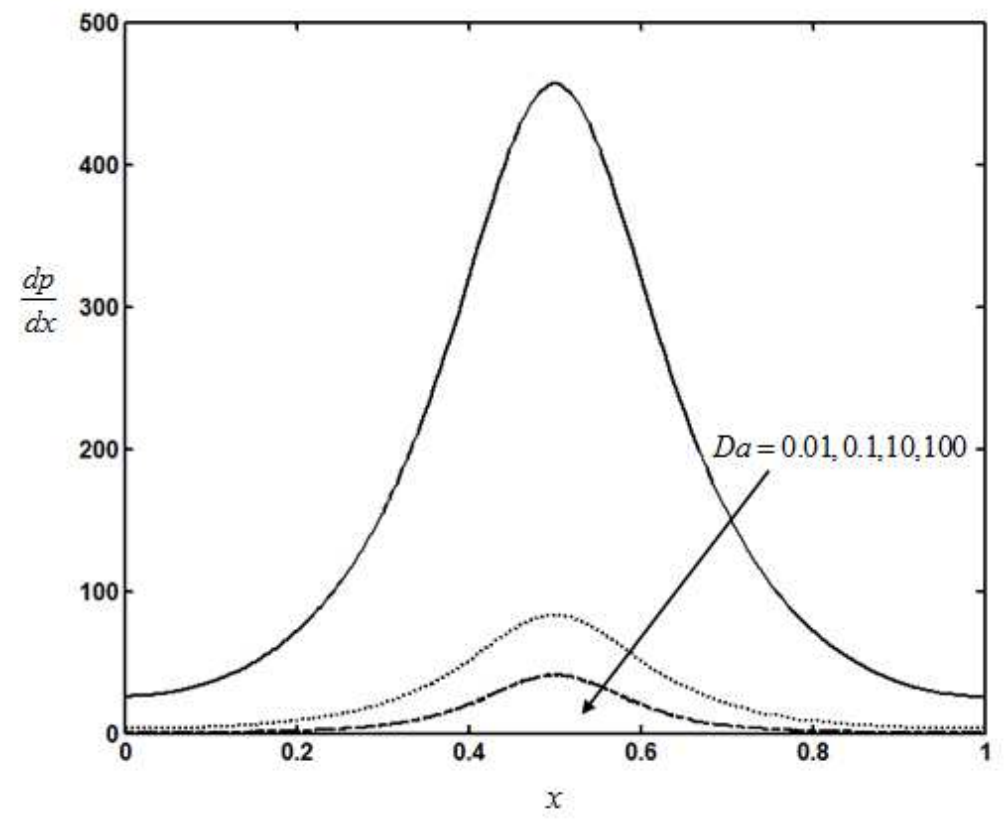

Figure 3: The Variation of Axial Pressure Gradient $\frac{d p}{d x}$ with

Darcy Number $D a$ for $\bar{Q}=-1, M=1, \phi=0.6, m=0.3, \beta=0.1$ and $\lambda_{1}=0.3$. 


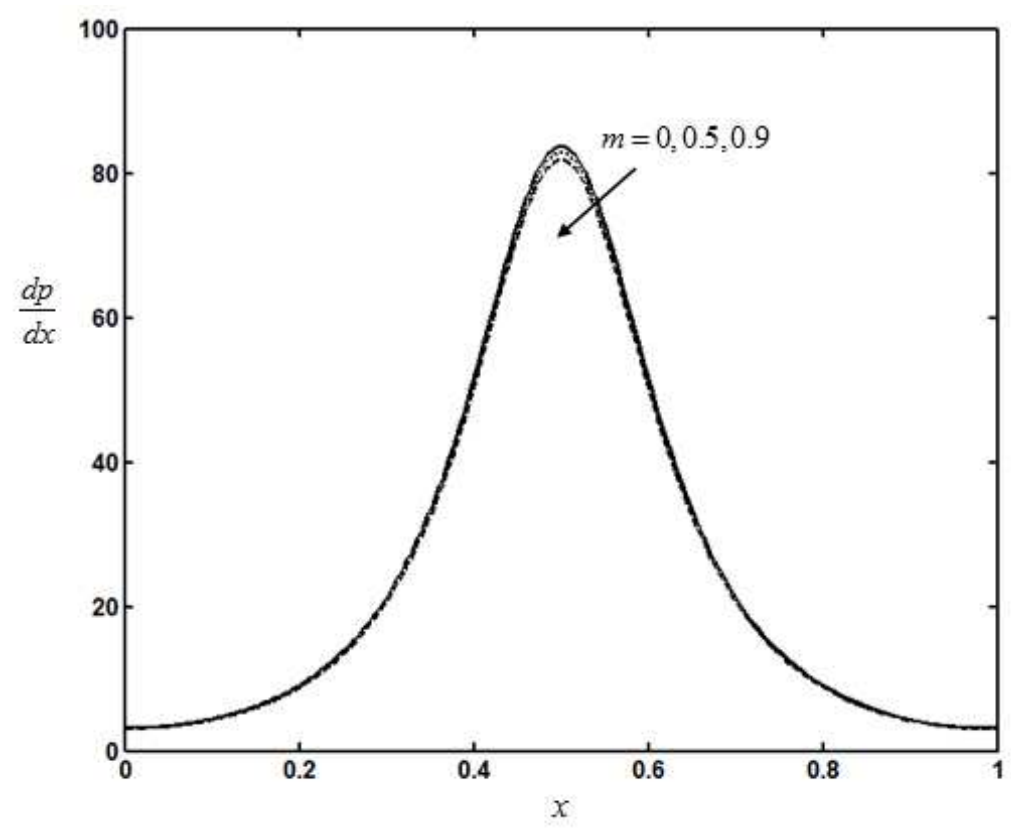

Figure 4: The Variation of Axial Pressure Gradient $\frac{d p}{d x}$ with Hall

Parameter $m$ for $\bar{Q}=-1, M=1, \phi=0.6, D a=0.1, \beta=0.1$ and $\lambda_{1}=0.3$.

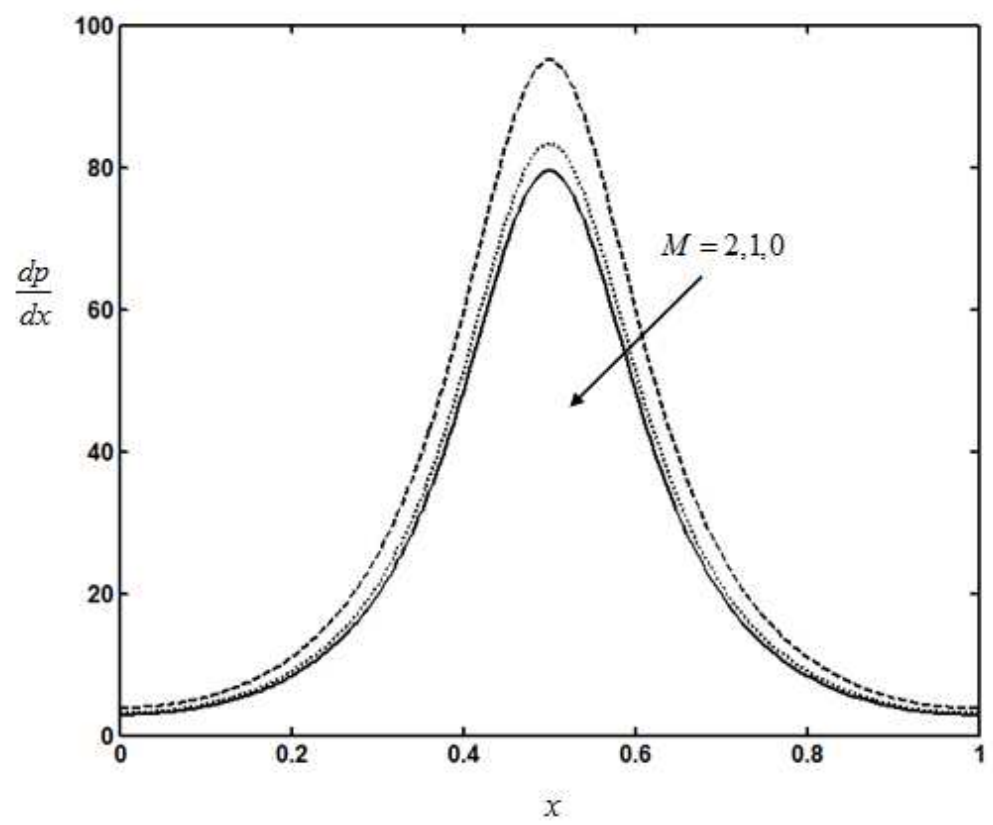

Figure 5: The Variation of Axial Pressure Gradient $\frac{d p}{d x}$ with Hartmann

Number $M$ for $\bar{Q}=-1, \lambda_{1}=0.3, D a=0.1, \phi=0.6, \beta=0.1$ and $m=0.3$. 


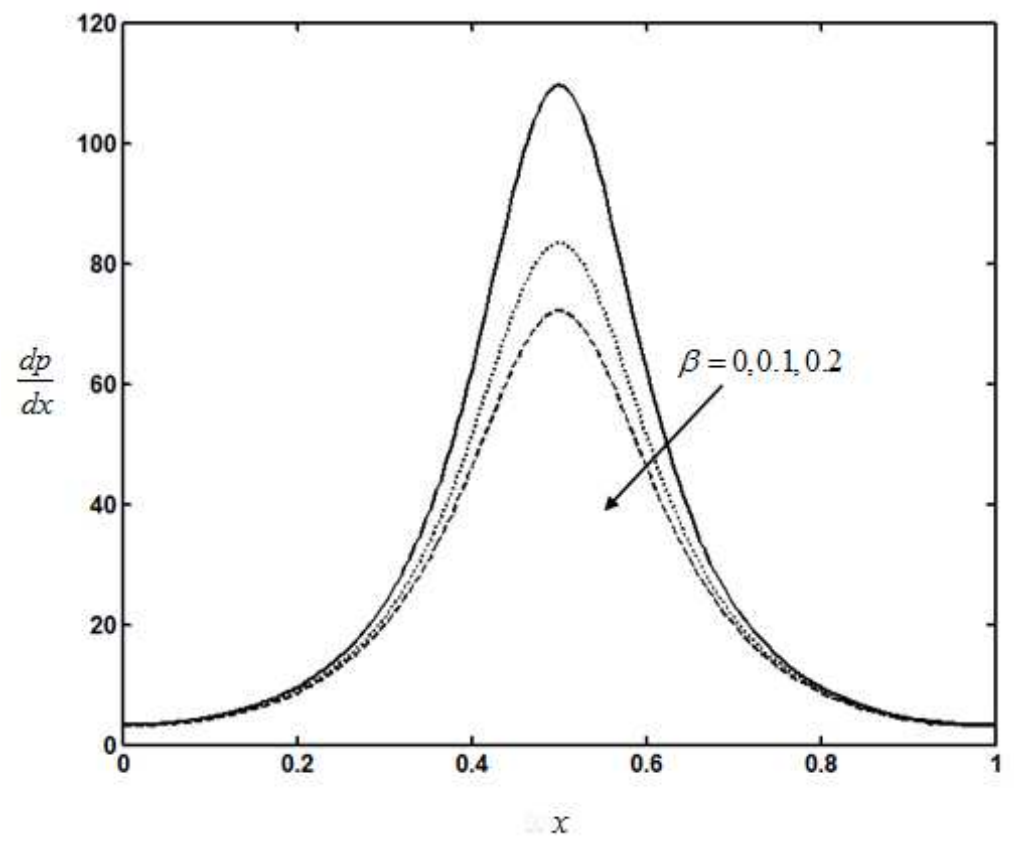

Figure 6: The Variation of Axial Pressure Gradient $\frac{d p}{d x}$ with Slip

Parameter $\beta$ for $\bar{Q}=-1, M=1, \phi=0.6, D a=0.1, \lambda_{1}=0.3$ and $m=0.3$.

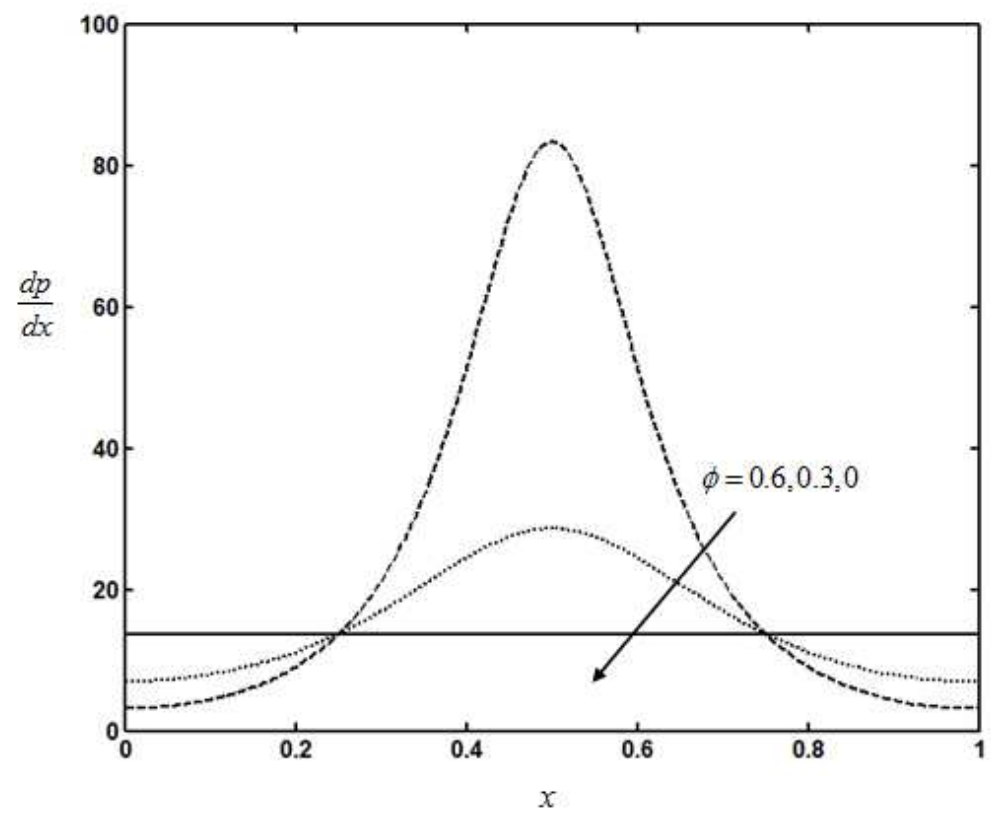

Figure 7: The Variation of Axial Pressure Gradient $\frac{d p}{d x}$ with Amplitude

Ratio $\phi$ for $\bar{Q}=-1, M=1, \lambda_{1}=0.3, D a=0.1, \beta=0.1$ and $m=0.3$. 


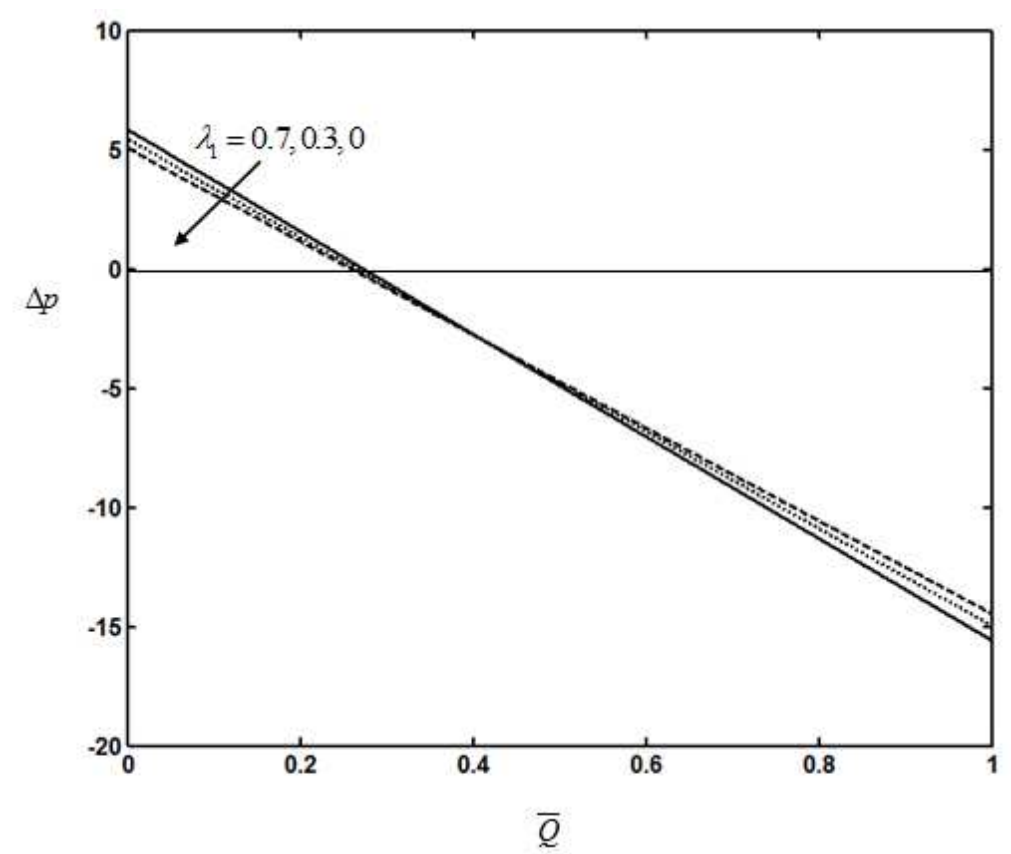

Figure 8: The Variation of Pressure Rise $\Delta p$ with Time-Averaged Flow Rate $\bar{Q}$ for Different Values of Jeffrey Fluid Parameter $\lambda_{1}$ with $M=1, D a=0.1, \phi=0.6, \beta=0.1$ and $m=0.3$.

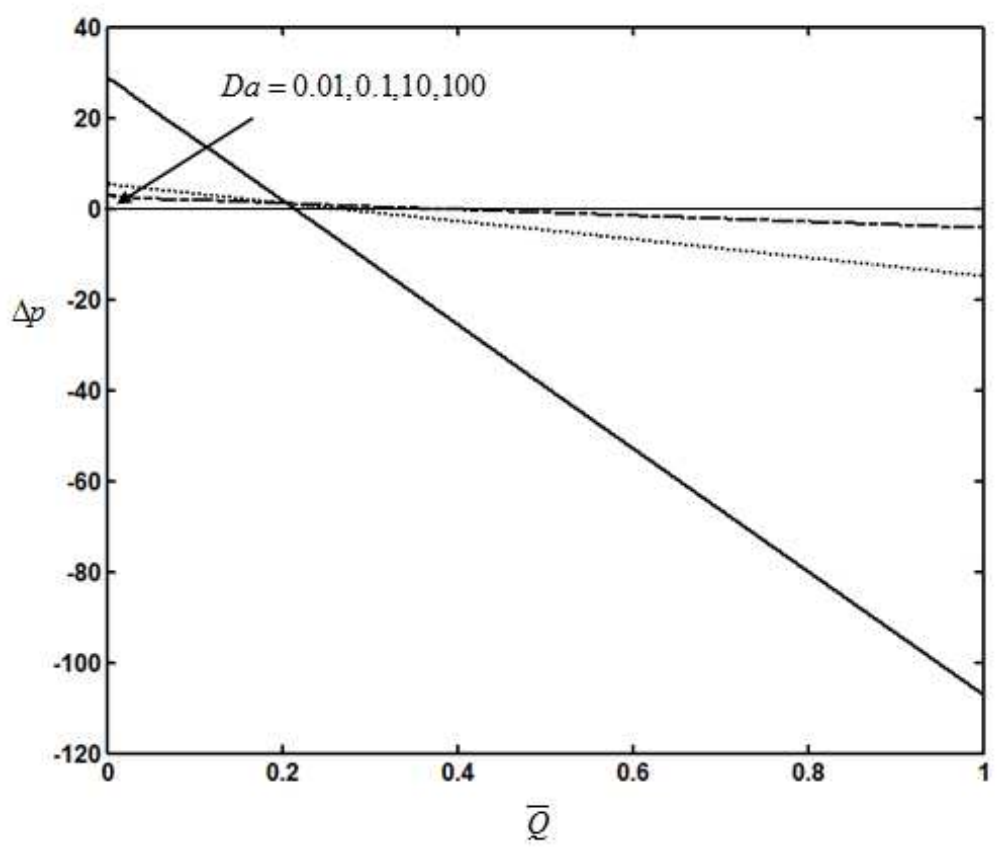

Figure 9: The Variation of Pressure Rise $\Delta p$ with Time-Averaged Flow Rate $\bar{Q}$ for Different Values of Darcy Number $D a$ with $M=1, \phi=0.6, m=0.3, \beta=0.1$ and $\lambda_{1}=0.3$. 


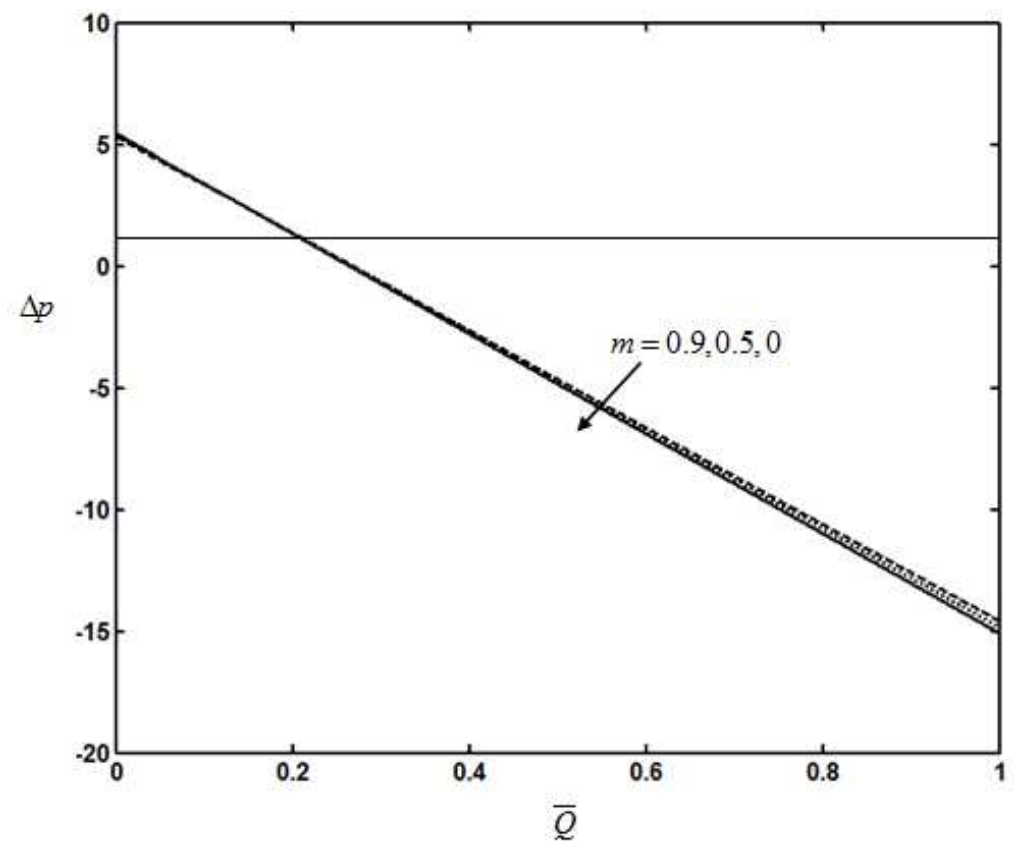

Figure 10: The Variation of Pressure rise $\Delta p$ with Time-Averaged Flow Rate $\bar{Q}$ for Different Values of Hall Parameter $m$ with $M=1, \phi=0.6, D a=0.1, \beta=0.1$ and $\lambda_{1}=0.3$.

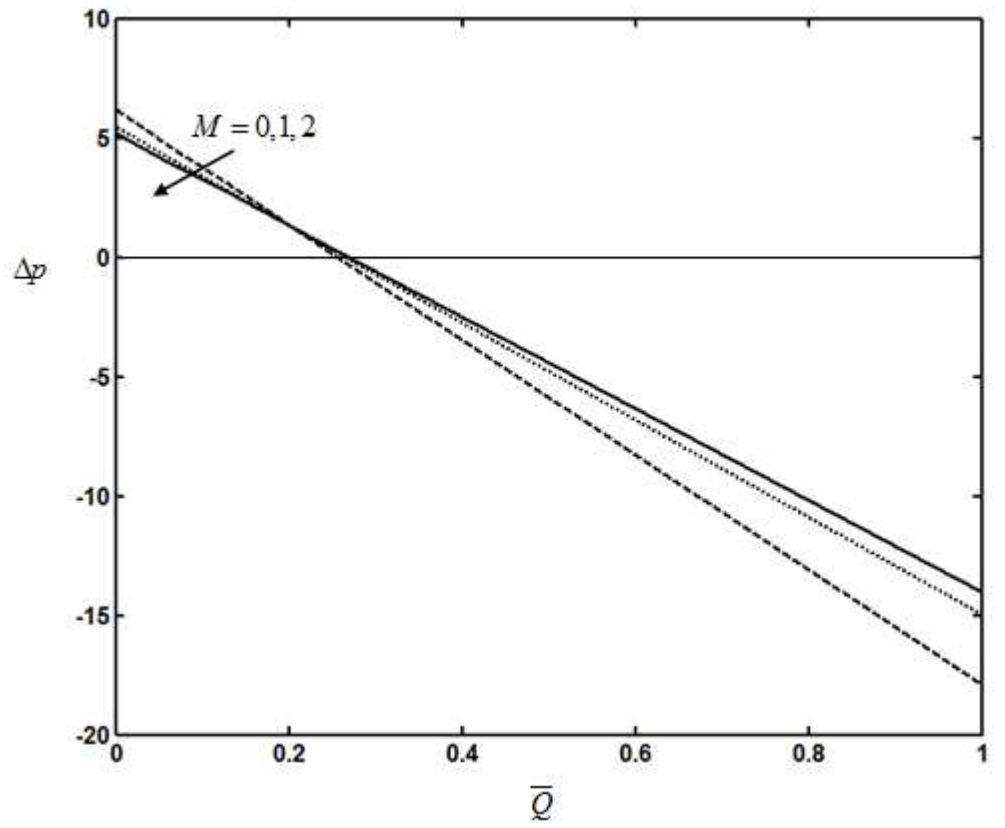

Figure 11: The Variation of Pressure Rise $\Delta p$ with Time-Averaged Flow Rate $\bar{Q}$ for Different Values of Hartmann Number $M$ with $\lambda_{1}=0.3, D a=0.1, \phi=0.6, \beta=0.1$ and $m=0.3$. 


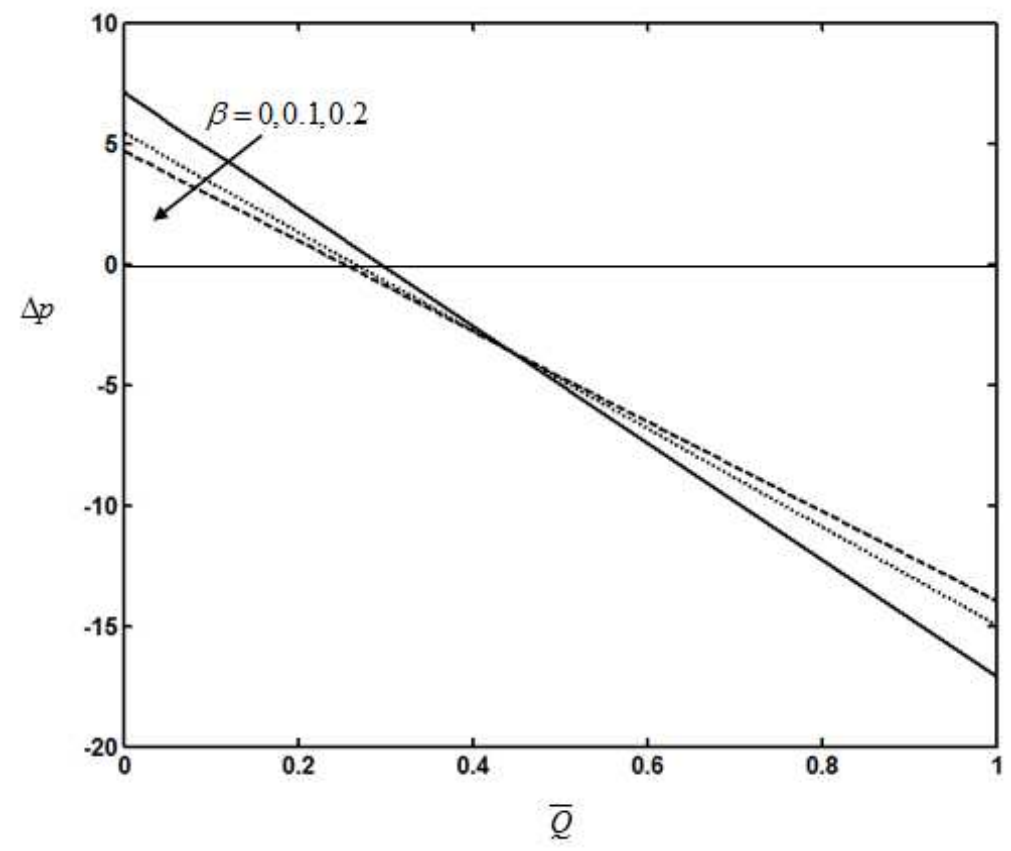

Figure 12: The Variation of Pressure Rise $\Delta p$ with Time-Averaged Flow Rate $\bar{Q}$ for Different Values of Slip Parameter $\beta$ with $M=1, \phi=0.6, D a=0.1, \lambda_{1}=0.3$ and $m=0.3$.

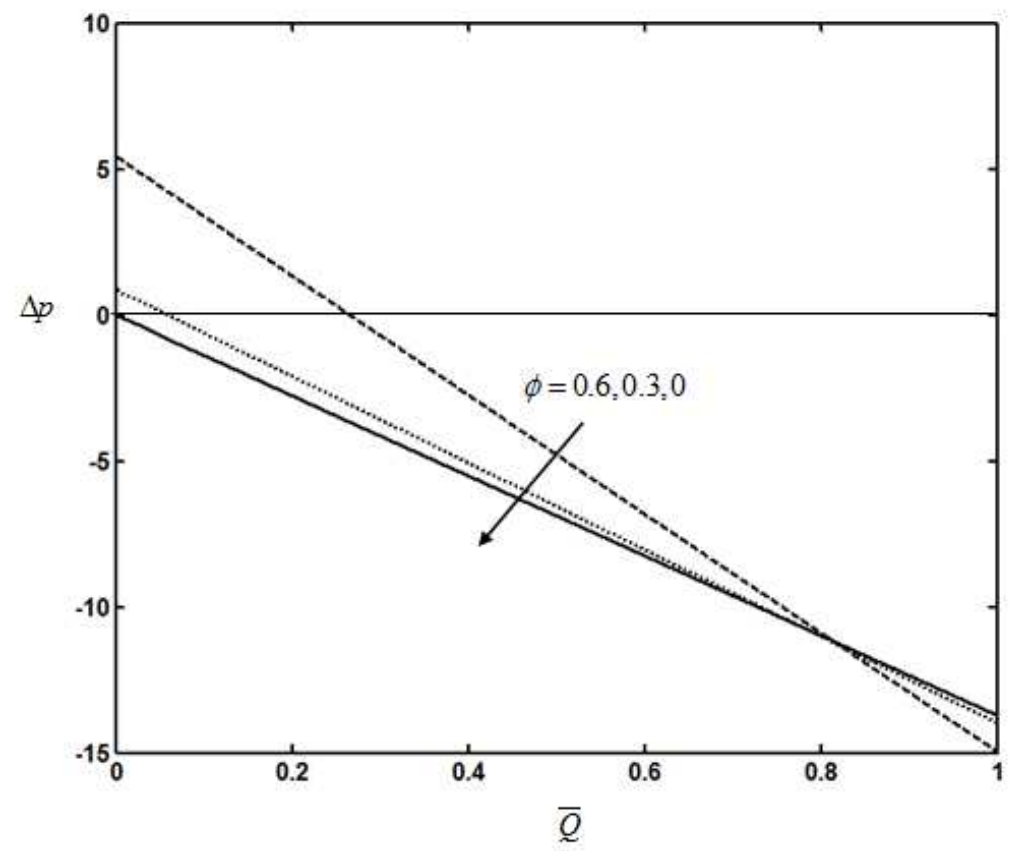

Figure 13: The Variation of Pressure Rise $\Delta p$ with Time-Averaged Flow Rate $\bar{Q}$ for Different Values of Amplitude Ratio $\phi$ with $M=1, \lambda_{1}=0.3, D a=0.1, \beta=0.1$ and $m=0.3$. 


\section{REFERENCES}

1. Abo-Eldahab, E.M., Barakat, E.I. and Nowar, K.I. (2010).Effects of Hall and ion-slip currents on peristaltic transport of a couple stress fluid, International Journal of Applied Mathematics and Physics, 2 (2), 145-157.

2. Ali, N., Wang, Y., Hayat, T. and Oberlack, M. (2009). Slip effects on the peristaltic flow of a third grade fluid in a circular cylindrical tube, J. Appl. Mech., 76, 1-10.

3. Al-Zubaidi, Diyaa HJ, Muhannad Al-Waily, and Emad Q. Hussein. "Analytical heat generation investigation for forced vibration beam with different crack characterizations influence." International Journal of Energy and Environment 10.1 (2019): 33-48.

4. Bhatti, M. M., Ali Abbas, M. and Rashidi, M. M. (2016).Effect of hall and ion slip on peristaltic blood flow of Eyring Powell fluid in a non-uniform porous channel, World Journal of Modelling and Simulation, 12 (4), 268-279.

5. Chaube, M.K., Pandey, S.K. and Tripathi, D. (2010).Slip Effect on Peristaltic Transport of Micropolar Fluid, Applied Mathematical Sciences, 4, 2105 - 2117.

6. Eldabe, N.T.M., Ahmed Y. Ghaly, A.Y., Sallam, S.N., Elagamy, K. and Younis, Y.M. (2015). Hall effect on peristaltic flow of third order fluid in a porous medium with heat and mass transfer, Journal of Applied Mathematics and Physics, 3, 1138-1150.

7. El-Shehawey, E.F., El-Dabe, N.T. and El-Desoky, I. M.(2006). Slip effects on the Peristaltic flow of a Non-Newtonian Maxwellian Fluid, Acta Mech., 186, 141-159.

8. Gad, N.S. (2014). Effects of Hall current on peristaltic transport with compliant walls, Appl Math Comput. 235, 546-554.

9. Hayat, T., Ali, N., Asghar, S. and Siddiqui, A. M. (2006).Exact peristaltic flow in tubes with an endoscope, Appl. Math.Comput. 182, 359-368.

10. Hayat, T., Ali, N. and Asghar, S. (2007). An analysis of peristaltic transport for flow of a Jeffrey fluid, ActaMechanica, 193, 101-112.

11. Hayat, T., Ali, N. and Asghar, S. (2007). Hall effects on peristaltic flow of a Maxwell fluid in a porous medium, Physics Letters A, 363, 397-403.

12. Hayat, A. and Ali, N. (2008).Peristaltic motion of a Jeffrey fluid under the effect of a magnetic field in a tube, Communications in Nonlinear Science and Numerical Simulation, 13, 1343-1352.

13. Hayat, T., Javed, M. and Ali, N. (2008).MHD peristaltic transport of a Jeffrey fluid in a channel with compliant walls and porous space, Transport in Porous Media 74(3), 259-274.

14. Jyothi, B., Satyanarayana, B. and Subba Reddy, M.V. (2015). Slip effects on peristaltic transport of a Prandtl fluid in a channel under the effect of magnetic field, South Asian Journal of Mathematics, 5(1), 1 -12.

15. Jyothi, S., Subba Reddy, M.V., Gangavathi, P. (2016). Hyperbolic tangent fluid flow through a porous medium in an inclined channel with peristalsis, International Journal of Advanced Scientific Research and Management, 1(4), 113-121.

16. Kwang - Hua Chu, W.and Fang, J. (2000). Peristaltic transport in a slip flow, Eur. Phys., J. B., 16, 543-547.

17. MekheimerKh.S. (2003).Non linear peristaltic transport through a porous medium in an inclined planar channel, J. Porous Media, 6, 190-202.

18. Noreen, S., Hayat, T.andAlsaedi, A. (2011). Study of slip and induced magnetic field on the peristaltic flow of pseudoplastic fluid, International Journal of Physical Sciences, 6(36), 8018-8026. 
19. Poonia, Hemant, and R. C. Chaudhary. "Mass transfer with chemical reaction effects on MHD free convective flow past an accelerated vertical plate embedded in a porous medium." Int. J. of Applied Mathematics \& Statistical Sciences 5 (2016).

20. Praveena, D., et al. "Unsteady Hydromagnitic Free Convective Heat Transfer Flow of Visco-Elasti C Fluid Through Porous Medium with Heat Source and Viscous Dissipation." International Journal of Mathematics and Computer Applications Research (IJMCAR) 5.6 (2015): 116 (2015).

21. Ranjitha, B. and Subba Reddy, M. V. (2018). Radiation effects on the peristaltic flow of a Jeffrey fluid through a porous medium in a channel, 2018, Int. J. Mathematical Archive, 9(9), 55-64.

22. Shapiro, A.H., Jaffrin, M.Y and Weinberg, S.L. (1969).Peristaltic pumping with long wavelengths at low Reynolds number, J. Fluid Mech. 37, 799-825.

23. SubbaNarasimhudu, K. andSubba Reddy, M.V. (2017). Hall effects on peristaltic pumping of a Newtonian fluid in a channel with long wavelength approximation, JUSPS-B, 29(6), 142-148.

24. Subba Reddy, M.V., Jayarami Reddy, B., Nagendra, N. and Swaroopa, B. (2012). Slip effects on the peristaltic motion of a Jeffrey fluid through a porous medium in an asymmetric channel under the effect magnetic field, Journal of Applied Mathematics and Fluid Mechanics, 4, 59-72.

25. Srihari, K., and ChirraKesava Reddy. "Effects of Soret and Magnetic Field on Unsteady Flow of a Radiating and Chemical Reacting Fluid: A Finite Difference Approach." International Journal of Mechanical Engineering 3.3 (2014).

26. Subba Reddy, M. V. andPrasanth Reddy, D. (2010). Peristaltic pumping of a Jeffrey fluid with variable viscosity through a porous medium in a planar channel, International Journal of Mathematical Archive,1(2), 42 - 54. 